\title{
Antithrombotic Medication and the Risk of Vitreous Hemorrhage in Atrial Fibrillation: Korean National Health Insurance Service National Cohort
}

\author{
Ko Eun Kim ${ }^{1 *}$, Pil-Sung Yang ${ }^{2 *}$, Eunsun Jang ${ }^{3}$, Sungjin Kim ${ }^{1}$, and Boyoung Joung ${ }^{3}$ \\ ${ }^{1}$ Department of Ophthalmology, Nowon Eulji Medical Center, Eulji University, Seoul; \\ ${ }^{2}$ Department of Cardiology, CHA Bundang Medical Center, CHA University, Seongnam; \\ ${ }^{3}$ Division of Cardiology, Yonsei University College of Medicine, Seoul, Korea.
}

Purpose: Antithrombotic therapy could be related with nuisance bleeding. This study investigated whether vitreous hemorrhage $(\mathrm{VH})$ is associated with specific types of antithrombotic medication in patients with atrial fibrillation (AF).

Materials and Methods: In the Korean National Health Insurance Service National Sample Cohort, we identified 9352 antiplatelet/ anticoagulant-treated AF patients. The occurrence of VH was compared between warfarin ( $\mathrm{n}=1493$ ) and a propensity score (PS)matched antiplatelet group $(\mathrm{n}=1493)$ and between warfarin $(\mathrm{n}=1493)$ and a PS-matched warfarin+antiplatelet group $(\mathrm{n}=1493)$.

Results: The outcomes of VH were lower in the warfarin than in the matched antiplatelet ( $1.45 \mathrm{vs.} 3.72$ events/1000 patient-years) and matched warfarin+antiplatelet groups (1.45 vs. 6.87 events/1000 patient-years). Compared with warfarin, the risk of VH increased with antiplatelet [adjusted hazard ratio (aHR) 3.90; 95\% confidence interval (CI) 1.22-12.4, $p=0.022$ ] and warfarin+antiplatelet agents (aHR 4.39, 95\% CI 1.74-11.2, $p=0.002$ ). Compared with warfarin only, warfarin+antiplatelet agents increased the risk of VH in patients $\geq 65$ years, regardless of gender and hypertension. The risk of $\mathrm{VH}$ was significantly higher with dual antiplatelet therapy (aHR: 5.02, 95\% CI: 1.56-16.2, $p=0.007$ ) or in dual (aHR: 5.02, 95\% CI: 1.74-14.5, $p=0.003$ ) or triple therapy using warfarin and antiplatelet agents than with warfarin monotherapy (aHR: 6.12, 95\% CI: 1.76-21.3, $p=0.004$ ).

Conclusion: Dual antiplatelet or triple therapy increased the risk of VH significantly, compared to warfarin monotherapy. Considering the low efficacy of preventing ischemic stroke and high risk of bleeding, dual or triple therapy using warfarin and antiplatelet agents should be avoided to prevent VH in AF patients.

Key Words: Vitreous hemorrhage, antiplatelet, anticoagulant, atrial fibrillation

\footnotetext{
Received: September 20, 2018 Revised: November 2, 2018

Accepted: November 19, 2018

Co-corresponding authors: Boyoung Joung, MD, PhD, Division of Cardiology, Severance Hospital, Yonsei University College of Medicine, 50-1 Yonsei-ro, Seodaemun-gu, Seoul 03722, Korea.

Tel: 82-2-2228-8460, Fax: 82-2-393-2041, E-mail: cby6908@yuhs.ac and Sungjin Kim, MD, PhD, Department of Ophthalmology, Nowon Eulji Medical Center, Eulji Univeristy, 68 Hangeulbiseok-ro, Nowon-gu, Seoul 01830, Korea.

Tel: 82-2-970-8322, Fax: 82-2-970-8003, E-mail: ksj4107@eulji.ac.kr

*Ko Eun Kim and Pil-Sung Yang contributed equally to this work.

-The authors have no potential conflicts of interest to disclose.

(C) Copyright: Yonsei University College of Medicine 2019

This is an Open Access article distributed under the terms of the Creative Commons Attribution Non-Commercial License (https://creativecommons.org/licenses/ by-nc/4.0) which permits unrestricted non-commercial use, distribution, and reproduction in any medium, provided the original work is properly cited.
}

\section{INTRODUCTION}

Atrial fibrillation (AF) is the most common cardiac arrhythmia, occurring in $1-2 \%$ of the general population. ${ }^{1,2} \mathrm{AF}$ confers a five-fold risk of stroke, and one in five of all strokes is attributed to this arrhythmia. ${ }^{3}$ Therefore, the administration of antithrombotic (antiplatelet or anticoagulant) medication is critical for the prevention of thromboembolic events in patients with AF. According to recent worldwide data, the number of patients taking antithrombotic medication is expected to rise with an increase in the number of AF patients. ${ }^{4}$ Although major bleeding, including intracranial hemorrhage or gastrointestinal bleeding, has been evaluated intensively, minor bleeding events (nuisance bleedings), including ocular bleeding, have not been well 
investigated. Recently, however, the importance of surveillance and adequate management for nuisance hemorrhagic complications arising from using these medications has been highlighted. ${ }^{5}$ Nuisance bleedings is common among patients with AF on oral anticoagulants (OACs). However, nuisance bleedings was not associated with a higher risk of major bleeding or stroke/systemic embolism over the next 6 months, suggesting its occurrence should not lead to changes in anticoagulation treatment strategies.

Vitreous hemorrhage $(\mathrm{VH})$ is a potential intraocular hemorrhagic complication associated with oral antiplatelet or anticoagulant medication, such as aspirin, clopidogrel bisulfate, and warfarin sodium. ${ }^{6-12}$ Although VH can be absorbed spontaneously, it can also result in more serious complications threatening vision, such as intraocular pressure increase and proliferative vitreoretinopathy, leading to invasive surgical intervention. Moreover, many AF patients discontinue antiplatelet or anticoagulant medication after $\mathrm{VH}$ and might experience thromboembolic events during this period. Therefore, the association between $\mathrm{VH}$ and antiplatelet or anticoagulant medication is important for both ophthalmologists and cardiologists.

Previous clinical studies have reported varying degrees of associations between $\mathrm{VH}$ and oral antiplatelet or anticoagulant use. ${ }^{6-12}$ However, most studies were performed on populations with specific retinal diseases or patients undergoing intraocular interventions. Epidemiological data are limited for generalizing the impact of antiplatelet or anticoagulation medication on $\mathrm{VH}$ risk. In this regard, we investigated the effects of antiplatelet and anticoagulation medication on VH development and non-pharmacological risk factors of $\mathrm{VH}$ in patients with $\mathrm{AF}$ using the Korean National Health Insurance Service (NHIS) database.

\section{MATERIALS AND METHODS}

\section{Data source}

This study was conducted using the NHIS-National Sample Cohort (NSC). ${ }^{13}$ The NHIS database represents the nationwide population of South Korea. ${ }^{1-3}$ The NHIS is a single insurer managed by the Korean government that covers $97 \%$ of the entire population; other programs, such the Medical Assistance Program, cover the remaining 3\%. The National Health Insurance Claim database stores data on the entire Korean population, including all demographic and medical information. Based on this database, the NHIS-NSC was created and released in $2014 .{ }^{13}$ It contains medical claims for $2.2 \%(n=1025340)$ of the total Korean population (about 50 million); individuals were randomly selected from January 1, 2002. NHIS-NSC data provide information on healthcare utilization based on the National Health Insurance claims from medical institutions to the NHIS for inpatient and outpatient clinic visits. ${ }^{1-3}$ The database is open to researchers once their study protocol has been approved by an official review committee. The Institutional Review Board of Severance Hospital at Yonsei University College of Medicine approved our study (4-2014-0996). Since we used anonymous data, the requirement to obtain informed consent was waived.

\section{Study population}

The current study included patients aged 19 years or older who were diagnosed with AF from January 2002 to December 2008. Patients with AF were identified using the International Classification of Disease, Tenth Revision (ICD-10): I480, I481, I482, I484, and I489. AF was validated in previous studies with a positive predictive value of $94.1 \% .^{1-3}$ To ensure diagnostic accuracy, we defined patients with AF only when they had a discharge diagnosis or were confirmed more than twice in the outpatient department. ${ }^{1-3}$ We excluded patients without a history of antiplatelet/anticoagulant use before enrollment. Among 9352 patients who were followed from January 2009 to December 2013, we identified 6074 antiplatelet agent-treated patients, 1493 warfarin-treated patients, and 1785 warfarin+antiplatelet agenttreated patients. The occurrence of $\mathrm{VH}$ was compared between warfarin ( $\mathrm{n}=1493)$ and a 1:1 propensity score (PS)-matched antiplatelet group $(n=1493)$ and between warfarin $(n=1493)$ and a 1:1 PS-matched warfarin+antiplatelet group (n=1493) (Fig. 1).

We obtained information on selected comorbid conditions from inpatient and outpatient hospital diagnoses. Baseline comorbidities were defined using the medical claims and prescription medication prior to the index date. In order to ensure diagnostic accuracy, patients were considered to have comorbidities when the condition was a discharge diagnosis or was

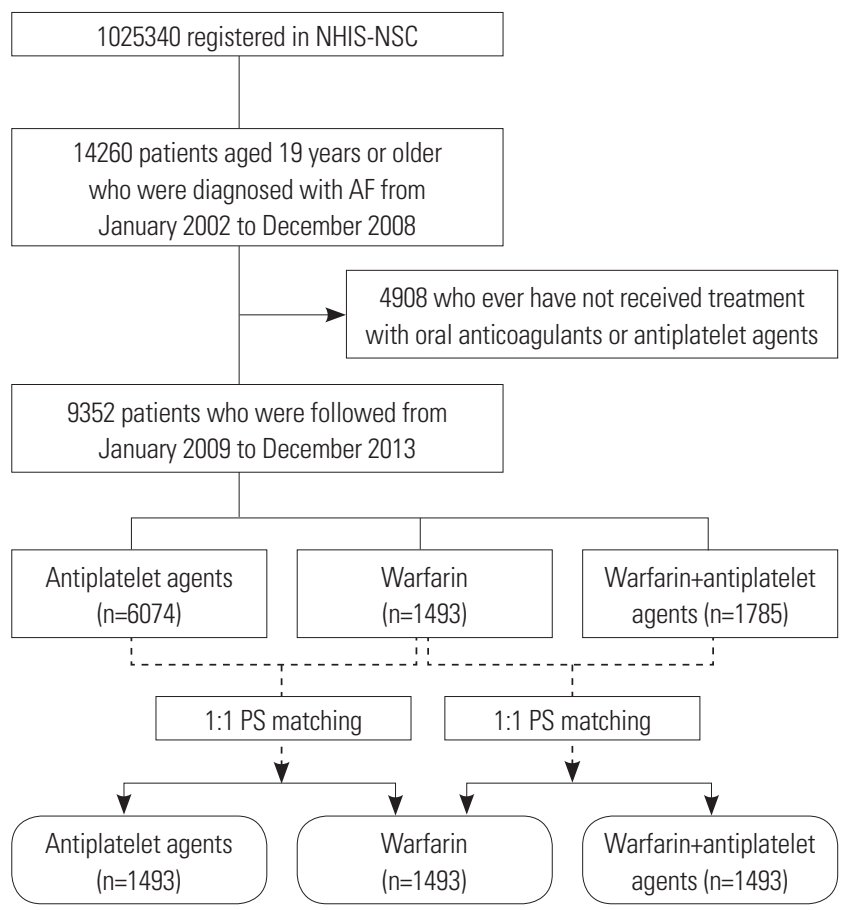

Fig. 1. Study cohort. NHIS-NSC, National Health Insurance Service-National Sample Cohort. AF, atrial fibrillation; PS, propensity score. 
confirmed at least twice in an outpatient setting, which was similar to previous studies with NHIS (Supplementary Table 1, only online). ${ }^{14-17}$ Also $\mathrm{CHA}_{2} \mathrm{DS}_{2}$-VASc [congestive heart failure, hypertension, age $\geq 75$ (doubled), diabetes mellitus, prior stroke or transient ischemic attack (TIA) (doubled), vascular disease, age 65 to 74, female] score and HAS-BLED [hypertension, abnormal renal/liver function (1 point each), stroke, bleeding history, labile INR (left out because of data are unavailable), elderly ( $>65$ years), concomitant drug ( 1 point each)] score were evaluated. ${ }^{1}$

\section{Exposure of antiplatelet and anticoagulation agents}

The exposure to antiplatelet and anticoagulation agents was identified based on filled antiplatelet or anticoagulant medication prescriptions over the follow-up period between January 2009 and December 2013. We used the most common medications for stroke prevention in patients with AF: aspirin and P2Y12 inhibitors (clopidogrel bisulfate, prasugrel, and ticagrelor) for antiplatelet medication and warfarin sodium for anticoagulation medication. The index date was defined as the date of first prescription of antiplatelet, warfarin, and warfarin + antiplatelet agents in antiplatelet, warfarin, and warfarin + antiplatelet groups, respectively.

\section{Study outcome}

The study outcome was newly developed VH (ICD-10 code H43.1) during the follow-up period between 2009 and 2013. To evaluate the accuracy of our definition of $\mathrm{VH}$, we conducted a validation study with 102 randomly chosen patients with the ICD-10 code H43.1. Fundus photos and ultrasonographic images were reviewed by two ophthalmologists (KEK and SJK). The patients were deemed to have $\mathrm{VH}$ if it was documented by fundus photos and ultrasonography examinations. The positive predictive value was $95.3 \%$. To ensure diagnostic accuracy, we defined patients with $\mathrm{VH}$ only when they had a discharge diagnosis or were confirmed more than twice in the outpatient department. The follow-up period was defined as from the index date until the first occurrence of $\mathrm{VH}$ or the end date of the study period, whichever came first.

\section{Statistical analysis}

The PS method, which simulates the effect of a randomized clinical trial for observational cohort data, was used to study the effects of warfarin on the occurrence of $\mathrm{VH}$ in comparison to other groups. PS reflects the predicted probability of treatment conditional on selected covariates, including age, sex, baseline comorbidities, and $\mathrm{CHA}_{2} \mathrm{DS}_{2}$-VASc and HAS-BELD scores, using logistic regression. The balance of covariates at baseline among the study groups was assessed using the absolute standardized mean difference (SMD). An absolute SMD $<0.1$ indicates a negligible difference in potential confounders between two study groups.

Incidence rates were estimated using the total number of study outcomes during the follow-up period divided by personyears at risk. The $95 \%$ confidence interval (CI) of incidence rate was estimated using a Poisson distribution. ${ }^{18}$ The risk of VH for warfarin versus other drugs was obtained using survival analysis: Kaplan-Meier method and log-rank test for univariate analysis and Cox proportional hazards regression for multivariate analysis. We adjusted for age, gender, heart failure, hypertension, diabetes, stroke/TIA, previous myocardial infarction, dyslipidemia, hemorrhagic stroke, valvular heart disease, malignancy, nonsteroidal anti-inflammatory drugs (NSAID), and corticosteroid medications. Statistical analyses were performed using Statistical Package for the Social Science version 19.0 for Windows (IBM Corp., Armonk, NY, USA) and MedCalc (v. 12.0; MedCalc Statistical software, Marakierke, Belgium). Statistical significance was defined as $p<0.05$.

\section{RESULTS}

\section{Study subjects}

Before PS matching, the warfarin group was younger, had lower $\mathrm{CHA}_{2} \mathrm{DS}_{2}$-VASc score, and had a lower proportion of comorbidities than other groups (Supplementary Table 2, only online). After PS matching, both of the two groups were well balanced with the warfarin group for all characteristics (all SMD $<0.1$ ). The warfarin group included more patients with valvular heart disease, including mitral stenosis or prosthetic mitral valve, than the other groups $(20.4,1.1$, and $7.0 \%, p<0.001)$ (Table 1 ).

\section{Primary study outcomes}

The cumulative incidence of $\mathrm{VH}$ is shown in Fig. 2. Overall, cumulative incidence curves revealed a lower rate of $\mathrm{VH}$ with warfarin than in matched antiplatelet ( $\log \operatorname{rank} p=0.003)$ (Fig. 2A) or warfarin+antiplatelet groups (log rank $p<0.001$ ) (Fig. 2B). The cumulative incidence of $\mathrm{VH}$ in each group is shown in Supplementary Fig. 1 (only online).

The outcomes of VH were lower for warfarin than for matched antiplatelet (1.45 vs. 3.72 events/ 1000 patient-years) and matched warfarin+antiplatelet groups (1.45 vs. 6.87 events/1000 patient-years). Compared with warfarin, the risk of $\mathrm{VH}$ was increased with antiplatelet [adjusted hazard ratio (aHR) 3.90; 95\% CI 1.22-12.4, $p=0.022]$ and warfarin+antiplatelet agents (aHR 4.39, 95\% CI 1.74-11.2, $p=0.002$ ) (Table 2).

Similar findings were observed in a sensitivity analysis that compared rates of $\mathrm{VH}$ after excluding patients with valvular heart disease. In sensitivity analysis, the outcomes of $\mathrm{VH}$ were lower in the warfarin than in the matched antiplatelet (1.79 vs. 5.33 events/1000 patient-years) and matched warfarin + antiplatelet groups ( 1.79 vs. 7.21 events/1000 patient-years). The risk of $\mathrm{VH}$ was lower for warfarin than for antiplatelet (aHR 4.64, 95\% CI 1.38-15.5, $p=0.013)$ and warfarin+antiplatelet groups (aHR 4.15, 95\% CI 1.57-11.0, $p=0.004$ ) (Table 3). 
Table 1. Characteristics of Warfarin and Propensity Score-Matched Antiplatelet or Warfarin+Antiplatelet-Treated Patients with Atrial Fibrillation

\begin{tabular}{|c|c|c|c|c|c|}
\hline & $\begin{array}{l}\text { Warfarin } \\
(n=1493)\end{array}$ & $\begin{array}{c}\text { Antiplatelet } \\
(n=1493)\end{array}$ & $\begin{array}{l}\text { Warfarin+antiplatelet } \\
\qquad(n=1493)\end{array}$ & $\begin{array}{c}\text { SMD } \\
\text { (warfarin vs. } \\
\text { antiplatelet) }\end{array}$ & $\begin{array}{c}\text { SMD } \\
\text { (warfarin vs. warfarin+ } \\
\text { antiplatelet) }\end{array}$ \\
\hline Age (yr) & $67(56,73)$ & $66(56,74)$ & $67(57,74)$ & -0.020 & -0.098 \\
\hline Men & 782 (52.4) & 798 (53.4) & $829(55.5)$ & 0.022 & 0.063 \\
\hline Congestive heart failure & $559(37.4)$ & $539(36.1)$ & $516(34.6)$ & 0.028 & 0.060 \\
\hline Hypertension & 1087 (72.8) & $1057(70.8)$ & $1141(76.4)$ & 0.045 & -0.081 \\
\hline Diabetes mellitus & $231(15.5)$ & $228(15.3)$ & $289(19.4)$ & 0.006 & -0.097 \\
\hline Ischemic stroke or TIA & $333(23.1)$ & 356 (23.8) & $345(23.1)$ & 0.019 & -0.018 \\
\hline Previous MI & $113(7.6)$ & $129(8.6)$ & $113(9.8)$ & 0.035 & -0.041 \\
\hline PAD & $104(7.0)$ & $104(7.0)$ & $131(8.8)$ & 0.049 & -0.077 \\
\hline Dyslipidemia & $639(42.8)$ & $626(41.9)$ & $717(48.0)$ & 0.018 & -0.096 \\
\hline Hemorrhagic stroke & $23(1.5)$ & $14(0.9)$ & $27(1.8)$ & 0.049 & -0.022 \\
\hline Malignancy & $233(15.6)$ & $215(14.4)$ & $238(15.9)$ & 0.032 & -0.009 \\
\hline $\mathrm{CHA}_{2} \mathrm{DS}_{2}$-VASc score & $3.00(2.00,4.00)$ & $3.0(1.0,4.0)$ & $3.0(2.0,5.0)$ & 0.032 & -0.067 \\
\hline HAS-BLED score & $2.0(1.0,3.0)$ & $2.0(1.0,3.0)$ & $3.0(2.0,3.0)$ & 0.011 & -0.091 \\
\hline \multicolumn{6}{|c|}{ Propensity score-unmatched variables } \\
\hline Valvular heart disease & $305(20.4)$ & $16(1.1)$ & $104(7.0)$ & & \\
\hline NSAID & $586(39.2)$ & $721(48.3)$ & $676(45.3)$ & & \\
\hline Steroid & $122(8.2)$ & $148(9.9)$ & $154(10.3)$ & & \\
\hline
\end{tabular}

$\mathrm{CHA}_{2} \mathrm{DS}_{2}$-VASc, congestive heart failure, hypertension, age of $\geq 75$ years (doubled), diabetes mellitus, prior stroke or TIA (doubled), vascular disease, age of 65 to 74 years, female sex; HAS-BLED, hypertension, abnormal renal/liver function (1 point each), stroke, bleeding history, labile INR (left out because of data are unavailable), elderly (>65 years), concomitant drug (1 point each); MI, myocardial infarction; NSAID, nonsteroidal anti-inflammatory drugs; PAD, peripheral artery disease; TIA, transient ischemic attack; SMD, standardized mean difference.

Values are presented as numbers (\%) or medians (interquartile range).

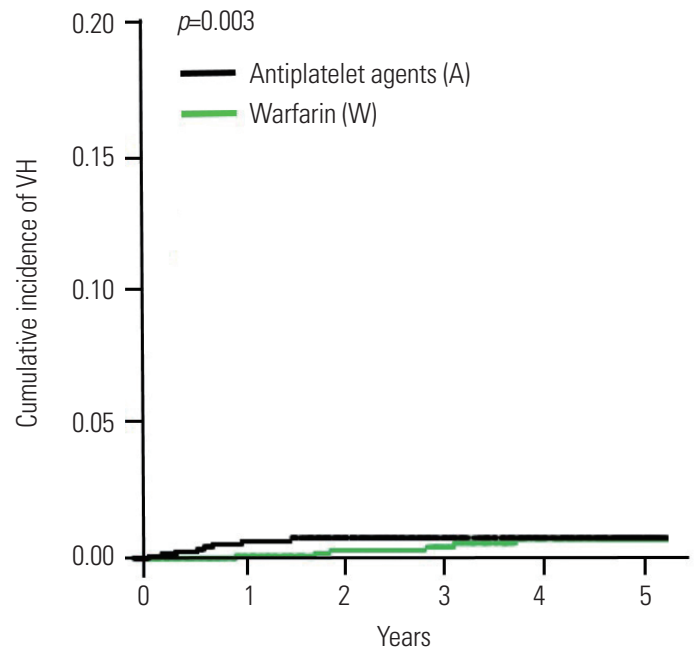

Number at risk

$\begin{array}{lrrrrrr}\text { A } & 1493 & 947 & 701 & 495 & 326 & 76 \\ \text { W } & 1493 & 1090 & 927 & 774 & 633 & 363\end{array}$

A

Fig. 2 Cumulative incidence of vitreous hemorrhage (VH). (A) Warfarin vs. an

\section{Outcomes according to different medications}

Compared with warfarin, the risk of $\mathrm{VH}$ was significantly higher in dual antiplatelet therapy using aspirin and P2Y2 inhibitor (1.45 vs. 8.70 events/1000 patient-years; aHR: $5.02,95 \%$ CI: 1.56-16.2, $p=0.007$ ), dual therapy using warfarin and aspirin (1.45 vs. 6.82 events/1000 patient-years; aHR: $5.02,95 \%$ CI:

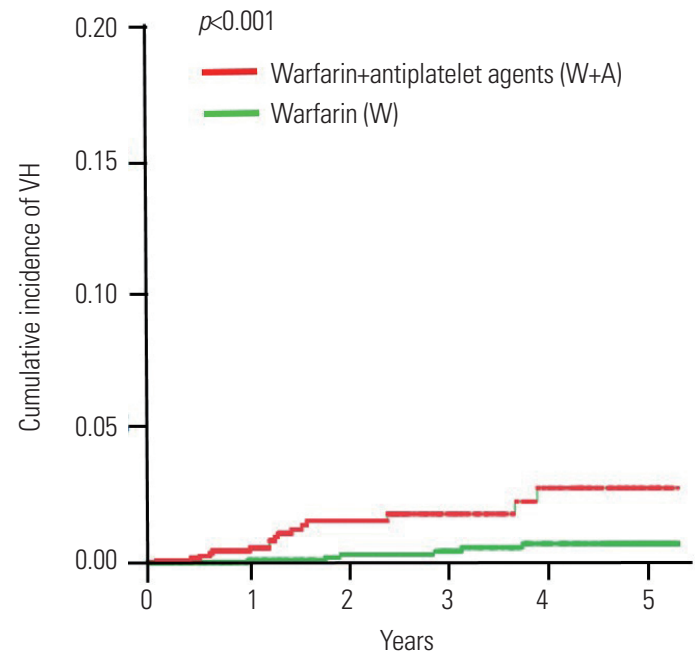

Number at risk

\begin{tabular}{|c|c|c|c|c|}
\hline$N+A$ & 1493 & 841 & 464 & 269 \\
\hline$W$ & 1493 & 1090 & 927 & 774 \\
\hline
\end{tabular}

B
1.74-14.5, $p=0.003$ ), and also triple therapy using warfarin, aspirin, and P2Y2 inhibitor (1.45 vs. 8.91 events/1000 patientyears; aHR: 6.12, 95\% CI: 1.76-21.3, $p=0.004)$. However, P2Y2 inhibitor monotherapy or dual therapy using warfarin and P2Y2 inhibitors did not increase the risk of VH (Fig. 3).

Supplementary Fig. 2 (only online) shows the risk of $\mathrm{VH}$ ac- 
cording to warfarin, antiplatelet agents, and warfarin + antiplatelet agents in overall patients.

\section{Outcomes by different subgroups}

Compared with warfarin, antiplatelet agents significantly increased the risk of $\mathrm{VH}$ in patients with hypertension and stroke or TIA (Fig. 4A). The risk of VH was generally increased by warfarin+antiplatelet agents across most subgroups, except younger age, diabetes, heart failure, and previous history of stroke or TIA. The risk of VH was increased in patients equal or older than 65 years, but not in those younger than 65 years (Fig. 4B).

Compared with warfarin, the risk of $\mathrm{VH}$ was generally increased by dual antiplatelet agents, dual therapy warfarin and
P2Y2 inhibitor, and triple therapy with warfarin, aspirin and P2Y2 inhibitor (Supplementary Fig. 3, only online).

\section{DISCUSSION}

The main findings of this study are a lower incidence of $\mathrm{VH}$ for warfarin than for antiplatelet or warfarin+antiplatelet treated individuals. Compared with warfarin, the risk of VH was increased with antiplatelet and warfarin+antiplatelet agents by 3.9 and 4.39 times, respectively. Second, compared with warfarin monotherapy, warfarin+antiplatelet agents increased the risk of $\mathrm{VH}$ in patients equal or more than 65 years, but not in those younger than 65 years. Finally, while combination treatment including aspirin (dual antiplatelet therapy, dual or

Table 2. Cox Proportional HR of the Risk of Different Types of Antiplatelet and Anticoagulant Medications for Vitreous Hemorrhage in Patients with Atrial Fibrillation

\begin{tabular}{|c|c|c|c|}
\hline & Warfarin (n=1493) & Antiplatelet agents ( $n=1493$ ) & Warfarin+antiplatelet agents ( $n=1493$ ) \\
\hline Events & 7 & 12 & 18 \\
\hline Person years & 4844 & 3230 & 2619 \\
\hline /1000 PYS (95\% CI) & $1.45(0.63-2.86)$ & $3.72(2.01-6.32)$ & $6.87(4.20-10.7)$ \\
\hline \multicolumn{4}{|l|}{ HR (95\% Cl), $p$ value } \\
\hline Unadjusted & 1 (Reference) & $5.11(1.59-16.4), 0.002$ & $5.19(2.11-12.7),<0.001$ \\
\hline Clinical variable-adjusted $^{*}$ & 1 (Reference) & $3.90(1.22-12.4), 0.022$ & $4.39(1.74-11.2), 0.002$ \\
\hline
\end{tabular}

$\mathrm{Cl}$, confidence interval; HR, hazard ratio; PYs, person years.

*Adjusted by age, gender, heart failure, hypertension, diabetes, stroke/transient ischemic attack, previous myocardial infarction, dyslipidemia, hemorrhagic stroke, valvular heart disease, malignancy, nonsteroidal anti-inflammatory drugs and corticosteroid medications.

Table 3. Cox Proportional HR of the Risk of Different Types of Antiplatelet and Anticoagulant Medications for Vitreous Hemorrhage in Patients with Non-Valvular Atrial Fibrillation

\begin{tabular}{|c|c|c|c|}
\hline & Warfarin $(n=1188)$ & Antiplatelet agents ( $n=1474$ ) & Warfarin+antiplatelet agents ( $n=1389$ ) \\
\hline Events & 6 & 17 & 17 \\
\hline Person years & 3355 & 3192 & 2357 \\
\hline /1000 PYs (95\% Cl) & $1.79(0.72-3.72)$ & $5.33(3.20-8.35)$ & $7.21(4.34-11.31)$ \\
\hline \multicolumn{4}{|l|}{ HR (95\% CI), $p$ value } \\
\hline Unadjusted & 1 (Reference) & 4.58 (1.37-15.3), 0.013 & 4.17 (1.62-10.8), 0.003 \\
\hline Clinical variable-adjusted ${ }^{*}$ & 1 (Reference) & 4.64 (1.38-15.5), 0.013 & $4.15(1.57-11.0), 0.004$ \\
\hline
\end{tabular}

$\mathrm{Cl}$, confidence interval; HR, hazard ratio; PYs, person years.

${ }^{*}$ Adjusted by age, gender, heart failure, hypertension, diabetes, stroke/transient ischemic attack, previous myocardial infarction, dyslipidemia, hemorrhagic stroke, valvular heart disease, malignancy, nonsteroidal anti-inflammatory drugs and corticosteroid medications.

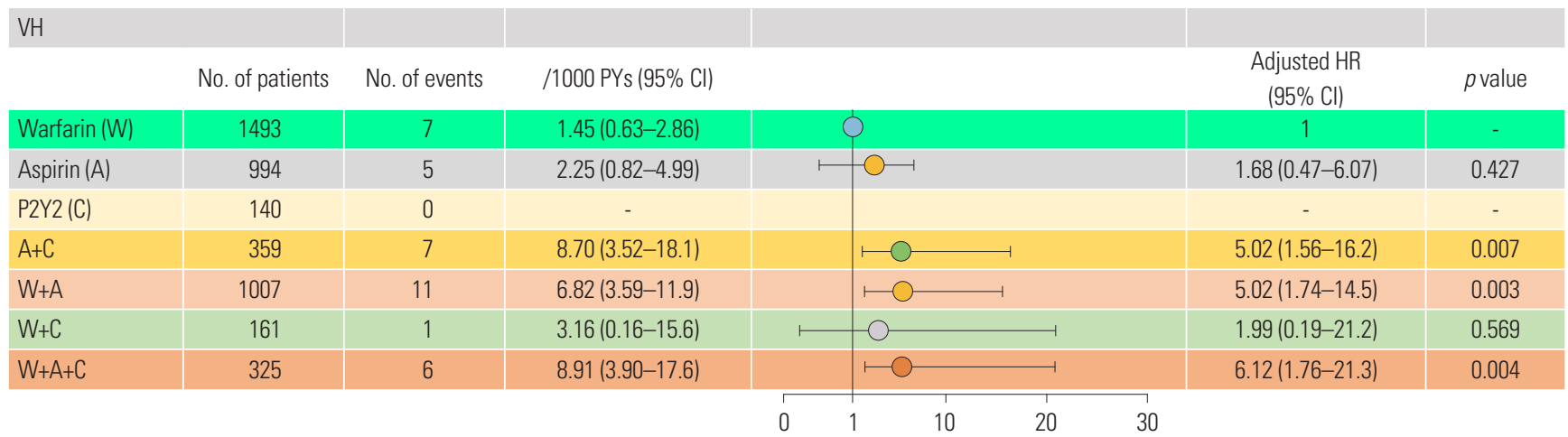

Fig. 3. Risk of VH according to different warfarin or antiplatelet agent combinations. VH, vitreous hemorrhage; $\mathrm{Cl}$, confidence interval; $\mathrm{HR}$, hazard ratio. 


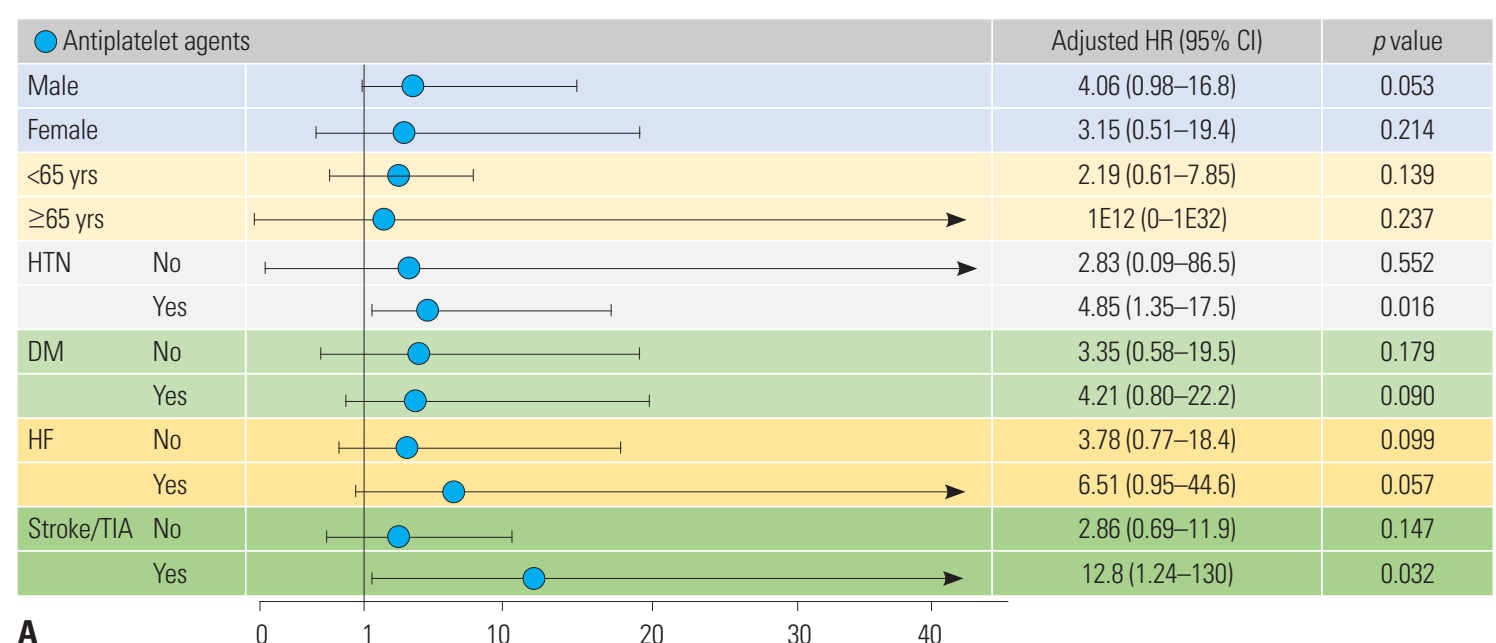

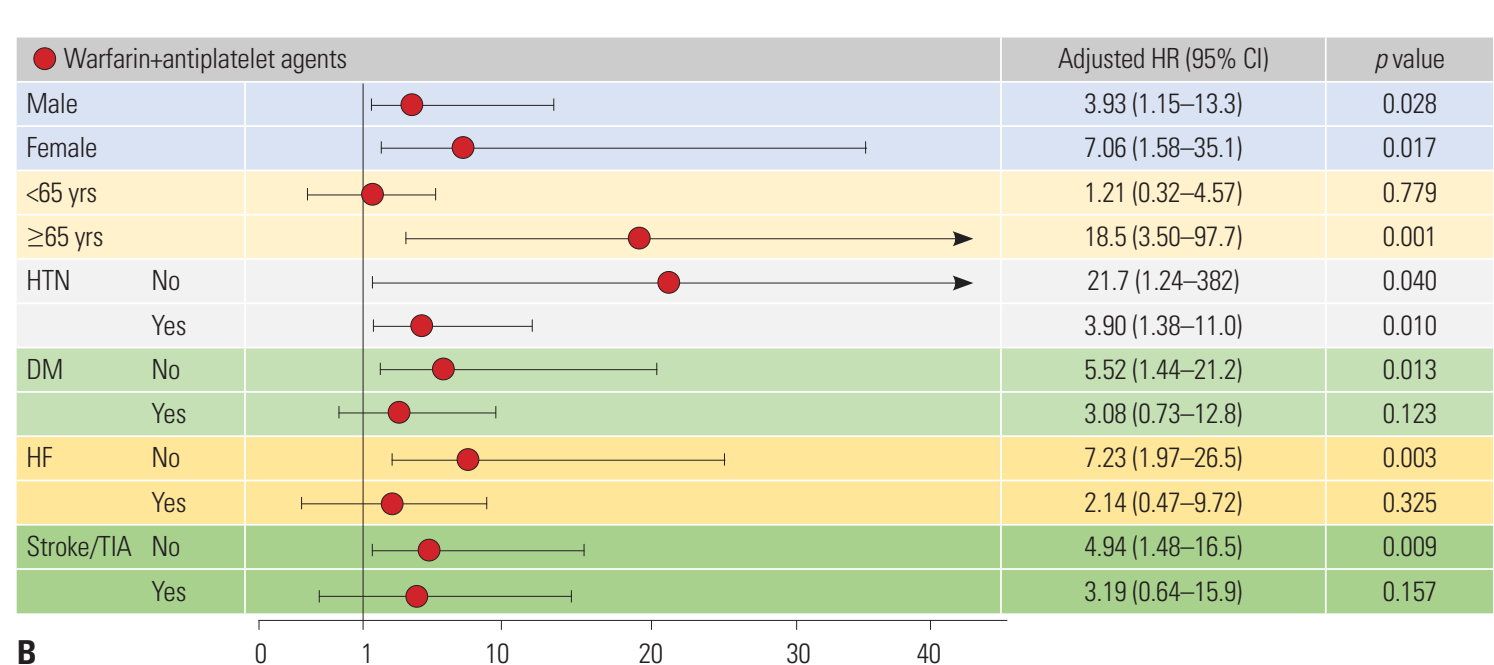

Fig. 4. Subpopulation analysis in patients with diverse comorbidities. The point estimates of vitreous hemorrhage risk in group with antiplatelet (A) or warfarin+antiplatelet combination (B) compared with reference (warfarin). HTN, hypertension; DM, diabetes mellitus; HF, heart failure; TIA, transient ischemic attack; $\mathrm{HR}$, hazard ratio; $\mathrm{Cl}$, confidence interval.

triple therapy using warfarin and antiplatelet agents) increased the risk of $\mathrm{VH}$, that without aspirin (dual therapy using warfarin and P2Y2 inhibitors) did not significantly increase the risk of $\mathrm{VH}$. This finding suggests that a combination of antiplatelet and anticoagulation agents should be avoided, especially in older adult patients, and cautiously recommends the use of P2Y2 inhibitor instead of aspirin if combination therapy is needed.

Stroke prevention is the principal management priority in patients with AF. Compared to control or placebo, OAC therapy reduces the risk of stroke by $64 \%$ and the risk of death by $26 \%,{ }^{19}$ but also increases bleeding risk, which can be fatal. Despite the positive net clinical benefit of OACs in the majority of patients with $\mathrm{AF}$, between $30 \%$ and $50 \%$ of patients meeting indications for OACs with $\mathrm{AF}$ are not on treatment. ${ }^{20}$ The OAC rate of total $\mathrm{AF}$ in the Korean nationwide cohort remains very low at about $18 \%$, while the usage rate of aspirin exceeds $35 \%{ }^{4,14}$ The analysis of a prospective multicenter study performed in tertiary hospitals in Korea [COmparison study of Drugs for symptom control and complication prEvention of Atrial Fibrillation (CODE-AF) registry] showed the optimistic future of stroke prevention in patients with AF. The current OAC rate of AF patients with high stroke risk $\left(\mathrm{CHA}_{2} \mathrm{DS}_{2}\right.$-VASc score $\left.\geq 2\right)$ was about $83 \%{ }^{21}$ Bleeding risk is commonly cited as a reason for stopping OAC therapy in high-risk patients. ${ }^{22,23}$

Nuisance bleeding events (e.g., bruising, epistaxis, intraocular bleeding) are substantially more common than more serious adverse bleeding events. ${ }^{24}$ As an indicator of bleeding tendency, they may influence decisions regarding discontinuation of OAC therapy, especially in older patients. ${ }^{25}$ Although it is considered a type of nuisance bleeding, intraocular hemorrhage is important and can lead to vision-threatening situations. The incidence of $\mathrm{VH}$ was reported as seven cases per 100000 person-years in the general population. ${ }^{26}$ However, the incidence of $\mathrm{VH}$ in $\mathrm{AF}$ patients with antithrombotic medications has not been well identified. While some studies have reported that antiplatelet or anticoagulation medication increases the possibility of intraocular hemorrhage ${ }^{6-8}$ others 
had contrary findings. ${ }^{9-12,27}$ In this study, while the incidence of VH was 1.45 cases per 1000 person-years in warfarin monotherapy, it increased to 8.91 cases per 1000 person-years by triple therapy. Dual antiplatelet therapy and dual therapy with aspirin and P2Y2 inhibitors significantly increased the risk of VH more than warfarin monotherapy. More importantly, aspirin monotherapy had an incidence of $\mathrm{VH}$ of 2.25 cases per 1000 person-years with the same bleeding risk as warfarin monotherapy. Therefore, considering the low efficacy of aspirin to prevent stroke and similar risk of bleeding, aspirin should be avoided in AF patients. However, the use of aspirin for stroke prevention in $\mathrm{AF}$ patients is more than $30 \%$ in Korean $\mathrm{AF}$ patients. ${ }^{14}$

Theoretically, triple therapy combining all drugs, including dual antiplatelet therapy and warfarin, may be a reasonable choice as an initial antithrombotic regimen. However, prolonged triple therapy has been shown to be associated with an increased risk of bleeding and even mortality. ${ }^{28,29}$ Recent well-designed RCTs suggested dual therapy with a single antiplatelet agent and an OAC might be safer and show similar efficacy to triple therapy for preventing ischemic/thromboembolic events. ${ }^{28,30-32}$ Dual therapy with clopidogrel and OAC was suggested to be a safe initial alternative regimen to triple therapy. ${ }^{30,31}$ Consistently, this study showed that dual therapy with warfarin and P2Y2 inhibitor did not significantly increase the risk of $\mathrm{VH}$.

Witmer and $\mathrm{Cohen}^{7}$ reported that patients taking aspirin, clopidogrel, or warfarin who developed acute posterior vitreous detachment were more likely to present with VH. Their results showed that the number of eyes with $\mathrm{VH}$ was significantly larger in patients taking antithrombotic medications than in those not taking them ( $43 \%$ vs. $31 \%, p=0.034$ ). Interestingly, P2Y2 inhibitors monotherapy or dual therapy using warfarin and P2Y2 inhibitors did not increase the risk of VH significantly.

Our study also showed that triple therapy increased the risk of $\mathrm{VH}$ in older adults equal or older than 65 years, but not in those younger than 65 years. Consistently, Biyik, et al. ${ }^{8}$ reported that warfarin increased the frequency of ocular bleeding in patients with hypertension and older age. In patients who were diagnosed as neovascular age-related macular degeneration (AMD), arterial hypertension was a strong risk factor for large subretinal hemorrhages, ${ }^{33}$ and antiplatelet or anticoagulant use was significantly associated with retinal or subretinal hemorrhage only in patients with hypertension. ${ }^{34}$ Previously, a significant association was reported between diabetes and hypertension with macular hemorrhage (including $\mathrm{VH}$ ) after intravitreal injection for neovascular AMD. ${ }^{35}$ Consistently, our findings suggest the importance of cautious monitoring for $\mathrm{VH}$ risk in patients with $\mathrm{AF}$ on stroke prevention therapy with such clinical characteristics.

Several limitations should be considered when interpreting our results. First, since the present study was a retrospective study using NHIS-NSC database, it had the intrinsic limitations as a retrospective study. Second, we tried to determine the incidence of spontaneous $\mathrm{VH}$ from antithrombotic medication; however, the ICD-10 code was not specialized to spontaneous $\mathrm{VH}$. It is possible that the incidence of $\mathrm{VH}$ is underestimated in patients who take anti-platelets/anti-coagulants based on database records, since the clinicians did not mention it in the medical record. However, because $\mathrm{VH}$ is a serious disease related with blindness, the possibility of underestimation is relatively low. Third, because the reason of discontinuation of anti-platelets/anti-coagulants was not available in the database, we could not present the number of patients who stopped taking anti-platelets/anti-coagulants due to VH. Finally, although we used PS matching among groups, uncontrolled covariates may exist. Despite these limitations, the strength of this study is that it included a large, population-based dataset to determine whether $\mathrm{VH}$ and the most commonly used antiplatelet and anticoagulation medications were associated.

In conclusion, dual antiplatelet or triple therapy including aspirin appears to increase the risk of $\mathrm{VH}$, compared to warfarin monotherapy. This finding suggests that a combination of antiplatelet and anticoagulation agents should be avoided, especially in elderly patients, and recommends the use of $\mathrm{P} 2 \mathrm{Y} 2$ inhibitor instead of aspirin if combination therapy is needed.

\section{ACKNOWLEDGEMENTS}

This research was supported by a grant from the Korean Healthcare technology R\&D project funded by the Ministry of Health \& Welfare (HI15C1200).

\section{ORCID iDs}

Ko Eun Kim Pil-Sung Yang Eunsun Jang Sungjin Kim Boyoung Joung

https://orcid.org/0000-0001-5125-7170

https://orcid.org/0000-0002-6552-1742 https://orcid.org/0000-0001-6991-4765 https://orcid.org/0000-0003-2474-9430 https://orcid.org/0000-0001-9036-7225

\section{REFERENCES}

1. Kim D, Yang PS, Jang E, Yu HT, Kim TH, Uhm JS, et al. 10-year nationwide trends of the incidence, prevalence, and adverse outcomes of non-valvular atrial fibrillation nationwide health insurance data covering the entire Korean population. Am Heart J 2018; 202:20-6.

2. Kim D, Yang PS, Jang E, Yu HT, Kim TH, Uhm JS, et al. Increasing trends in hospital care burden of atrial fibrillation in Korea, 2006 through 2015. Heart 2018;104:2010-7.

3. Kim TH, Yang PS, Uhm JS, Kim JY, Pak HN, Lee MH, et al. CHA2DS2-VASc score (congestive heart failure, hypertension, age $\geq 75$ [doubled], diabetes mellitus, prior stroke or transient ischemic attack [doubled], vascular disease, age 65-74, female) for stroke in Asian patients with atrial fibrillation: a Korean nationwide sample cohort study. Stroke 2017;48:1524-30.

4. Yang PS, Ryu S, Kim D, Jang E, Yu HT, Kim TH, et al. Variations of prevalence and incidence of atrial fibrillation and oral anticoagulation rate according to different analysis approaches. Sci Rep 2018;8:6856.

5. O'Brien EC, Holmes DN, Thomas LE, Fonarow GC, Allen LA, 
Gersh BJ, et al. Prognostic significance of nuisance bleeding in anticoagulated patients with atrial fibrillation. Circulation 2018;138: 889-97.

6. Kiernan DF, Hariprasad SM, Rusu IM, Mehta SV, Mieler WF, Jager $\mathrm{RD}$. Epidemiology of the association between anticoagulants and intraocular hemorrhage in patients with neovascular age-related macular degeneration. Retina 2010;30:1573-8.

7. Witmer MT, Cohen SM. Oral anticoagulation and the risk of vitreous hemorrhage and retinal tears in eyes with acute posterior vitreous detachment. Retina 2013;33:621-6.

8. Biyik I, Mercan I, Ergene O, Oto O. Ocular bleeding related to warfarin anticoagulation in patients with mechanical heart valve and atrial fibrillation. J Int Med Res 2007;35:143-9.

9. Brown JS, Mahmoud TH. Anticoagulation and clinically significant postoperative vitreous hemorrhage in diabetic vitrectomy. Retina 2011;31:1983-7.

10. Olson JM, Scott IU, Kerchner DL, Kunselman AR. Association between systemic anticoagulation and rate of intraocular hemorrhage following intravitreal anti-VEGF therapy for age-related macular degeneration. Ophthalmic Surg Lasers Imaging Retina 2013;44:455-9.

11. Benzimra JD, Johnston RL, Jaycock P, Galloway PH, Lambert G, Chung AK, et al. The Cataract National Dataset electronic multicentre audit of 55,567 operations: antiplatelet and anticoagulant medications. Eye (Lond) 2009;23:10-6.

12. Katz J, Feldman MA, Bass EB, Lubomski LH, Tielsch JM, Petty BG, et al. Risks and benefits of anticoagulant and antiplatelet medication use before cataract surgery. Ophthalmology 2003;110:1784-8.

13. Lee J, Lee JS, Park SH, Shin SA, Kim K. Cohort Profile: The National Health Insurance Service-National Sample Cohort (NHISNSC), South Korea. Int J Epidemiol 2017;46:e15.

14. Lee H, Kim TH, Baek YS, Uhm JS, Pak HN, Lee MH, et al. The trends of atrial fibrillation-related hospital visit and cost, treatment pattern and mortality in Korea: 10-year nationwide sample cohort data. Korean Circ J 2017;47:56-64.

15. Baek YS, Yang PS, Kim TH, Uhm JS, Park J, Pak HN, et al. Associations of abdominal obesity and new-onset atrial fibrillation in the general population. J Am Heart Assoc 2017;6:e004705.

16. Lee HY, Yang PS, Kim TH, Uhm JS, Pak HN, Lee MH, et al. Atrial fibrillation and the risk of myocardial infarction: a nation-wide propensity-matched study. Sci Rep 2017;7:12716.

17. Song S, Yang PS, Kim TH, Uhm JS, Pak HN, Lee MH, et al. Relation of chronic obstructive pulmonary disease to cardiovascular disease in the general population. Am J Cardiol 2017;120:1399-404.

18. Sahai H, Khurshid A. Statistics in epidemiology: methods, techniques, and applications. Boca Raton (FL): CRC Press; 1996.

19. Hart RG, Pearce LA, Aguilar MI. Meta-analysis: antithrombotic therapy to prevent stroke in patients who have nonvalvular atrial fibrillation. Ann Intern Med 2007;146:857-67.

20. Ogilvie IM, Newton N, Welner SA, Cowell W, Lip GY. Underuse of oral anticoagulants in atrial fibrillation: a systematic review. Am J Med 2010;123:638-45.

21. Kim H, Kim TH, Cha MJ, Lee JM, Park J, Park JK, et al. A prospective survey of atrial fibrillation management for real-world guideline adherence: COmparison study of Drugs for symptom control and complication prEvention of Atrial Fibrillation (CODE-AF)
Registry. Korean Circ J 2017;47:877-87.

22. O'Brien EC, Simon DN, Allen LA, Singer DE, Fonarow GC, Kowey $\mathrm{PR}$, et al. Reasons for warfarin discontinuation in the outcomes registry for better informed treatment of atrial fibrillation (ORBITAF). Am Heart J 2014;168:487-94.

23. Gumbinger C, Holstein T, Stock C, Rizos T, Horstmann S, Veltkamp R. Reasons underlying non-adherence to and discontinuation of anticoagulation in secondary stroke prevention among patients with atrial fibrillation. Eur Neurol 2015;73:184-91.

24. Altman R, Rivas AJ, Gonzalez CD. Bleeding tendency in dual antiplatelet therapy with aspirin/clopidogrel: rescue of the template bleeding time in a single-center prospective study. Thromb J 2012; 10:3.

25. Rosenman MB, Baker L, Jing Y, Makenbaeva D, Meissner B, Simon TA, et al. Why is warfarin underused for stroke prevention in atrial fibrillation? A detailed review of electronic medical records. Curr Med Res Opin 2012;28:1407-14.

26. Lindgren G, Sjödell L, Lindblom B. A prospective study of dense spontaneous vitreous hemorrhage. Am J Ophthalmol 1995;119: 458-65.

27. Oh J, Smiddy WE, Kim SS. Antiplatelet and anticoagulation therapy in vitreoretinal surgery. Am J Ophthalmol 2011;151:934-9.

28. Dewilde WJ, Oirbans T, Verheugt FW, Kelder JC, De Smet BJ, Herrman JP, et al. Use of clopidogrel with or without aspirin in patients taking oral anticoagulant therapy and undergoing percutaneous coronary intervention: an open-label, randomised, controlled trial. Lancet 2013;381:1107-15.

29. Rubboli A, Faxon DP, Juhani Airaksinen KE, Schlitt A, Marín F, Bhatt DL, et al. The optimal management of patients on oral anticoagulation undergoing coronary artery stenting. The 10th anniversary overview. Thromb Haemost 2014;112:1080-7.

30. Cannon CP, Bhatt DL, Oldgren J, Lip GYH, Ellis SG, Kimura T, et al. Dual antithrombotic therapy with dabigatran after PCI in atrial fibrillation. N Engl J Med 2017;377:1513-24.

31. Gibson CM, Mehran R, Bode C, Halperin J, Verheugt FW, Wildgoose $\mathrm{P}$, et al. Prevention of bleeding in patients with atrial fibrillation undergoing PCI. N Engl J Med 2016;375:2423-34.

32. Golwala HB, Cannon CP, Steg PG, Doros G, Qamar A, Ellis SG, et al. Safety and efficacy of dual vs. triple antithrombotic therapy in patients with atrial fibrillation following percutaneous coronary intervention: a systematic review and meta-analysis of randomized clinical trials. Eur Heart J 2018;39:1726-35a.

33. Kuhli-Hattenbach C, Fischer IB, Schalnus R, Hattenbach LO. Subretinal hemorrhages associated with age-related macular degeneration in patients receiving anticoagulation or antiplatelet therapy. Am J Ophthalmol 2010;149:316-21.

34. Ying GS, Maguire MG, Daniel E, Grunwald JE, Ahmed O, Martin DF; Comparison of Age-Related Macular Degeneration Treatments Trials Research Group. Association between antiplatelet or anticoagulant drugs and retinal or subretinal hemorrhage in the comparison of age-related macular degeneration treatments trials. Ophthalmology 2016;123:352-60.

35. Moon SW, Oh J, Yu HG, Cho HY, Song SJ. Incidence and risk factors for macular hemorrhage following intravitreal ranibizumab injection for neovascular age-related macular degeneration. J Ocul Pharmacol Ther 2013;29:556-9. 